\title{
Avaliação dos processos de fratura em filmes finos através da simulação de ensaios de indentação com penetradores esféricos
}

\section{Evaluation of fracture processes on thin films by simulation of spherical indentation testing}

Ozias Ferreira Chaves Filho ${ }^{1}$, Avelino Manuel da Silva Dias ${ }^{2}$

\author{
${ }^{1}$ Programa de Pós-graduação em Engenharia Mecânica, CT/UFRN CEP: 59064-741, Natal, RN, Brasil. \\ ${ }^{2}$ Departamento de Engenharia Mecânica, CT/UFRN CEP: 59064-741, Natal, RN, Brasil. \\ e-mail: oziasferr@gmail.com, avelino.dias@ct.ufrn.br
}

\section{RESUMO}

Recentes trabalhos propõem a utilização dos ensaios de indentação como uma ferramenta capaz de avaliar características mecânicas de filmes finos, assim como avaliar possíveis falhas em sistemas que conjugam filmes de alta dureza depositados em substratos metálicos em solicitações tribológicas. Entretanto, a implementação da técnica de indentação para a avaliação do comportamento destes sistemas filme/substrato e os seus resultados, continuam ocasionando dúvidas no meio científico. Em função destas incertezas na análise do ensaio de indentação, o uso da análise numérica capaz de avaliar os campos de tensões e de deformações durante o ciclo de indentação pode auxiliar em uma interpretação mais segura deste ensaio. Por isso, este trabalho tem como objetivo utilizar o Método dos Elementos Finitos $(M E F)$ para simular ensaios de indentação em um filme de Nitreto de Cromo e Alumínio $(\mathrm{Cr} A l N)$ de alta dureza depositado em um substrato de aço carbono. Foram usados dois diferentes modelos de danos para avaliar os processos de fratura que ocorrem nestes ensaios de indentação. O primeiro foi o modelo de trinca difusa e o segundo foi o modelo de delaminação, ambos presentes no software comercial de elementos finitos MARC ${ }^{\text {TM }}$. Os processos de fratura observados nestas simulações foram comparados com outros resultados apresentados na literatura especializada. Por fim, o uso concomitantemente dos dois modelos de danos foi uma nova abordagem para o estudo de falhas nos ensaios de indentação em filmes finos.

Palavras-chave: elementos finitos, modelo de trinca difusa, modelo de delaminação, mecânica da fratura, filmes finos.

\section{ABSTRACT}

Recent studies propose using indentation testing as a tool to assess the mechanical characteristics of thin films, as well as evaluate possible cracking in systems that conjugate films with high hardness values deposited on metallic substrates in tribological solicitations. However, implementing the indentation technique to assess the behavior of these film/substrate systems, and their results continue to raise doubts in the scientific community. Because of these uncertainties in the studies of the indentation testing, the use of numerical analysis capable of evaluating stress and strain fields during the indentation cycle may help better interpret this test. As such, this study aimed to use the finite element method (FEM) to simulate indentation testing of Chronium Aluminum Nitride ( $\mathrm{CrAlN}$ ) high-hardness film deposited in a carbon steel substrate. Two different damage models were used to assess the fracture processes that occur in these testing. The first was the diffuse cracking model and the second the delamination model, both present in MARCTM finite element software. The fracture processes observed in these simulations were compared with other published results. Finally, the concomitant use of these two damage models was a new approach to the study of indentation testing damage in thin films.

Keywords: finite elements, cracking strain, delamination model, fracture mechanics, thin films. 


\section{INTRODUÇÃO}

A procura por melhorias nas propriedades tribológicas de componentes tem impulsionado o avanço na área de engenharia de superfícies. Esta área trata da tecnologia de preparação e modificação das superfícies para cumprir funções específicas dentro de certa aplicação [1]. Atualmente, uma das opções mais utilizadas para melhorar estas propriedades é o uso de filmes cerâmicos finos, que, entre outras vantagens, pode melhorar a resistência ao desgaste. Contudo, para aperfeiçoar o uso desses recobrimentos, é necessário estudar as propriedades mecânicas destes, do substrato, assim como da interface formada entre o filme e o substrato. $\mathrm{O}$ ensaio de indentação instrumentada tem sido utilizado para avaliar as propriedades mecânicas destes sistemas [2-3].

Esses ensaios de indentação têm sido amplamente utilizados para a determinação da dureza superficial em diferentes classes de materiais. Contudo, devido à sua versatilidade, trabalhos foram desenvolvidos neste campo, avaliando novas metodologias e aplicações para este tipo de ensaio [2-4]. Por exemplo, alguns trabalhos propõem o uso dos ensaios de indentação para determinar o módulo de elasticidade $(E)$, coeficiente de Poisson $(v)$, a tenacidade à fratura $(K I C)$ e, mais recentemente, uma possível curva de tensão em função da deformação do comportamento elastoplástico sob compressão [3-6].

Entretanto, a implementação desta técnica de indentação encontram algumas dificuldades, ocasionando dúvidas no meio científico. Por exemplo, segundo a literatura, os ensaios apresentam problemas mais intensos quando se pretende avaliar o comportamento mecânico de filmes finos depositados em substratos macios [4]. Além disso, para a realização do ensaio de indentação instrumentada são necessários equipamentos de alta precisão capazes de aplicar e monitorar baixíssimas cargas, sendo sensíveis as deformações na amostra, utilizando grandezas em micro e em nano escalas [2-6]. Por isso, devido a estas limitações, o uso de uma técnica numérica confiável que seja capaz de simular o ciclo de indentação, permitindo avaliar os campos de tensões e de deformações gerados, pode auxiliar o estudo desses ensaios e apresentar uma interpretação mais segura deles [5-12].

Falhas em sistemas que usam recobrimentos de alta dureza com substratos de baixa dureza em serviço, durante solicitações tribológicas, raramente acontecem por desgaste convencional, mas podem acontecer principalmente na interface entre o recobrimento e o substrato (falha adesiva), ou fratura do recobrimento (falha coesiva) ou, ainda, por fratura sub-superficial (falha do substrato) dependendo de diversos fatores como tamanho do indentador, propriedades do recobrimento, entre outras [5, 9, 12].

Para estudar estas falhas de sistemas filmes/substratos torna-se essencial determinar a distribuição do campo de tensões que levam a ocorrência de deformações plásticas nestes conjugados, assim como determinar a região onde se dá o início e desenvolvimento desta zona plástica [5, 12]. Portanto, o uso de métodos numéricos que simulem o ciclo de indentação também seria útil para analisar falhas que podem ocorrer durante o processo. Neste sentido, a análise numérica via Método dos Elementos Finitos $(M E F)$ se mostra uma técnica bastante versátil, podendo ser usada em estudos nos mais variados campos, resolvendo problemas não lineares nas áreas de instabilidade estrutural, de sistemas dinâmicos, termodinâmicos, sistemas de mecânica da fratura, de conformação mecânica, entre outras [5-13].

Este trabalho tem como objetivo usar o Método dos Elementos Finitos ( $M E F)$ em conjunto com os conceitos da mecânica dos meios contínuos e da mecânica da fratura linear elástica (MFLE) para simular ensaios de indentação com penetradores esféricos e analisar processos de fratura que podem ocorrer durante esses ensaios através de dois modelos de danos, o modelo de trinca difusa e o modelo de delaminação [12, 14-15]. Finalmente, este trabalho comparou os resultados obtidos com os apresentados na literatura especializada, avaliando os resultados encontrados através dos dois modelos de dano adotados.

As simulações foram feitas considerando um filme fino de Nitreto de Cromo e Alumínio $(\mathrm{CrAlN})$ depositado em um substrato de aço carbono (AISI 4140). Filmes de nitretados exibiram um grande interesse na indústria devido à alta dureza, alto ponto de fusão e alta estabilidade química. No entanto, há um número crescente de aplicações onde às propriedades mecânicas e tribológicas destes filmes não são suficientes. Uma maneira de melhorá-las é a criação de filmes de composição complexas (nitretos ternários), ligando o nitreto binário com outro elemento, como $\operatorname{CrTiN}, \operatorname{CrAlN}, \mathrm{CrTiAlN}$, etc [16]. Destes, o $\mathrm{CrAlN}$ representa uma nova geração de revestimento baseado na liga de cromo que foi investigada por muitos autores [5, 6, 14-19]. A adição de alumínio afeta a estrutura do revestimento e, em seguida, aumenta os valores de módulo de elasticidade $(E)$ que estão relacionados com o percentual de alumínio. Assim, filmes $\mathrm{CrAlN}$ apresentam melhor desempenho antidesgaste e alta dureza para as amplas aplicações em potenciais de engenharia como, por exemplo, em ferramentas de corte e em dentes de engrenagens. Por isso, este sistema foi escolhido uma vez que o $C r A l N$ também apresenta boa adesão ao substrato, alta resistência ao desgaste, boa dureza e boa resistência a oxidação, ou seja, boas propriedades tribológicas [16-19]. 
Nas simulações realizadas, variou-se a espessura do filme $C r A l N$ e a profundidade de penetração do indentador. Por fim, realizou-se uma análise para o limiar de falhas neste revestimento estudado em função de sua espessura e da profundidade de penetração do indentador.

\section{MATERIAIS E MÉTODOS}

As simulações do ensaio de indentação foram feitas utilizando o software comercial de Elementos Finitos

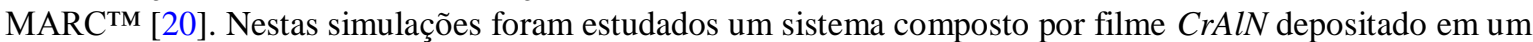
substrato de aço AISI 4140 através de um processo de deposição física a vapor $(P V D)$. As propriedades mecânicas utilizadas para simular este sistema filme/substrato estão mostradas na Tabela 1.

Tabela 1: Propriedades dos materiais que compõem o sistema estudado [5, 21].

\begin{tabular}{c|c|c|c|c|c}
\hline Material & $\boldsymbol{E}(\mathbf{G P a})$ & $\boldsymbol{v}$-Coeficiente de Poisson & $\boldsymbol{S y}$ - Limite de Escoamento (MPa) & $\boldsymbol{K}$ & $\boldsymbol{n}$ \\
\hline AISI 4140 & 238 & 0,29 & 565 & 2.230 & 0,228 \\
\hline Filme CrAlN & 350 & 0,22 & 3.790 & 10.615 & 0,229 \\
\hline
\end{tabular}

Nas simulações realizadas, tanto o filme como o substrato foram, considerados como materiais isotrópicos, homogêneos e com um comportamento elasto-plástico definido pela clássica expressão descrita na literatura e mostrada na Equação (1) $[6,15,21]$. Nesta, $\sigma$ é a tensão efetiva, $E$ é o módulo de elasticidade do material, $\varepsilon$ é a deformação efetiva do material, enquanto $K$ e $n$ são constantes que descrevem o encruamento do material denominadas coeficiente de resistência e coeficiente de encruamento, respectivamente.

$$
\sigma=\left\{\begin{array}{l}
E \epsilon^{1.0}(\text { elástico }) \\
K \epsilon^{n}(\text { plástico })
\end{array}\right.
$$

O sistema filme/substrato foi modelado através de elementos axis-simétricos de quatro nós, com o intuito de diminuir o tempo de simulação e, consequentemente, reduzir seu custo computacional. O indentador esférico com diâmetro de $400 \mu \mathrm{m}(R=200 \mu \mathrm{m})$, foi simulado através de uma casca circular rígida que penetra na amostra dos sistemas a serem estudados, Figura 1. Como condições de contorno do problema, o modelo possui restrição ao deslocamento na sua base e restrição ao deslocamento radial nos nós que estão localizados no seu eixo de simetria.
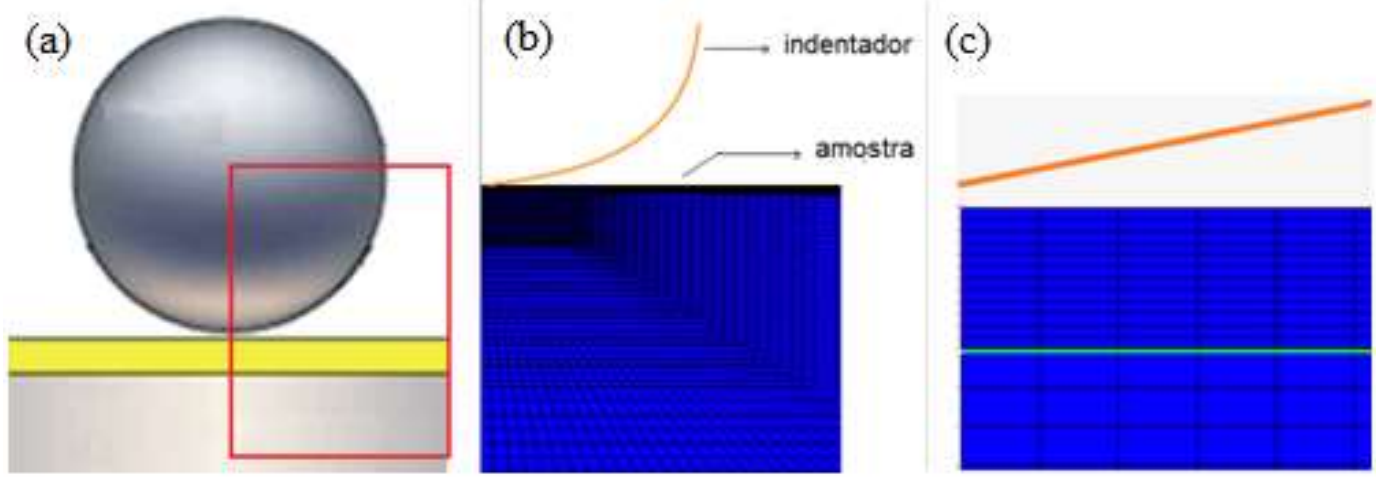

Figura 1: (a) Ilustração do ensaio de dureza com indentador esférico [14]; (b) Malha utilizada nas simulações deste ensaio usando elementos axis-simétricos; (c) ampliação da malha na região de contato do indentador com a amostra.

Simulou-se o ciclo de indentação, carregamento e descarregamento do ensaio, através do deslocamento prescrito do indentador, permitindo-se, assim, um melhor controle numérico no início e durante a simulação. Desconsiderou-se o coeficiente de atrito entre o indentador e a amostra, pois, conforme literatura especializada, o atrito não influencia significativamente nos resultados numéricos de carga de indentação [7, 14].

Foram adotadas três diferentes combinações dos dois modelos de dano utilizados. Na primeira, foi simulado o ensaio de indentação utilizando apenas o modelo de trinca difusa [12]. Na segunda, foi implementado apenas o modelo de delaminação na interface entre o filme e o substrato [15]. Na terceira combinação, 
foram utilizados estes dois modelos. Ou seja, aplicou-se o modelo de trinca difusa para estudar o comportamento do filme e, para modelar a interface entre o filme e o substrato, utilizou-se elementos de delaminação. Essa última combinação é uma nova proposta para a simulação de processos de fratura em ensaios de indentação, aproveitando das vantagens destes dois modelos de danos.

Nos ensaios estudados, foram feitas simulações com as seguintes espessuras do filme de CrAlN: 2,0 $\mu \mathrm{m}, 3,0 \mu \mathrm{m}, 6,0 \mu \mathrm{m}$ e 9,0 $\mu \mathrm{m}$. Para cada espessura do filme, foram feitos cinco ensaios onde foi variada a profundidade de penetração, sendo iguais a: $10 \%$ da espessura do filme, 20\%, 50\%, $70 \%$ e 100\%. Em baixas profundidades de penetração (10\%), os campos de tensões e de deformação se distribuem fortemente no filme $[5,19]$. Para grandes profundidades de penetração (50 a 100\%), o substrato se mostra significativamente afetado, assim como os resultados da dureza do sistema [9, 14, 21-22]. Assim, o uso desta faixa de profundidades de penetração permitiu uma análise mais abrangente do comportamento do campo de tensões e dos processos de fratura em toda amostra. Estes valores de profundidade de penetração também foram baseados em outros trabalhos publicados da mesma área, permitindo, assim, fazer uma comparação entre estes trabalhos $[9,12,14,21]$.

O filme estudado $(C r A l N)$ apresenta um comportamento que pode ser simulado pelo modelo de trinca difusa, ou seja, é um material frágil que apresenta baixa resistência à tração, porém possui boa resistência à compressão, podendo, inclusive, sofrer deformações plásticas, sob compressão. Neste trabalho, o parâmetro de bimodularidade (Es) utilizado no modelo de trinca difusa foi estimado em $10 \%$ do módulo de elasticidade (E) do CrAlN. Esta estimativa foi baseada nos estudos encontrados na literatura especializada, em especial nos trabalhos de Dias et. al. (2010) e Medeiros et. al. (2012), que estudaram os processos de fratura em filmes finos e em materiais frágeis, respectivamente $[12,23]$.

$\mathrm{Na}$ interface entre o filme e o substrato foram introduzidos os elementos de delaminação (Figura 1C) para ativar o modelo de delaminação. Este modelo de dano utiliza uma lei de tração-separação através da estimativa do valor crítico da energia coesiva $\left(\mathrm{G}_{\mathrm{IC}}\right)$ do filme [15, 20, 24]. Após se atingir o valor crítico desta energia coesiva, os nós dos elementos de delaminação começam a se separar. O procedimento adotado para se encontrar uma estimativa desta energia coesiva está descrito a seguir.

\subsection{Estimativa do valor da Energia Coesiva do CrAIN}

Uma vez que não foi encontrada na literatura especializada o valor da tenacidade à fratura $\left(K_{I C}\right)$ do filme $C r A l N$, estimou-se seu valor utilizando simulações do ensaio de mecânica da fratura com corpos de prova compacto CT [23-27]. Nesta etapa, reproduziu-se a metodologia desenvolvida por Chaves Filho et. al. (2015) capaz de estimar a tenacidade à fratura em diferentes materiais frágeis [27]. Nessa metodologia foi feito um modelo numérico do ensaio $\mathrm{CT}$, de modo que as suas dimensões atendessem às exigências estabelecidas pela norma ASTM E 399 (1984). As dimensões deste corpo de prova para o filme CrAlN estão mostradas na Tabela 2 .

Tabela 2: Dimensões do corpo de prova CT simulado.

\begin{tabular}{l|l|l|l}
\hline$H C(\mathrm{~mm})$ & $W(\mathrm{~mm})$ & $a(\mathrm{~mm})$ & $B(\mathrm{~mm})$ \\
\hline 15,0 & 25,0 & 12,5 & 12,5 \\
\hline
\end{tabular}

Para simular o ensaio com corpo de prova compacto, a malha resultante apresentou a configuração mostrada na Figura 2. Esta malha representou apenas a parte superior do corpo de prova, considerando a simetria do problema e, consequentemente, economizando esforço computacional. Após a determinação da tenacidade a fratura do $C r A l N$, utilizou-se a Equação (2) para estimar o valor do $G_{I C}$ do filme, considerando os conceitos da mecânica da fratura linear elástica [23-27].

$$
G_{I C}=\frac{K_{I C}^{2}\left(1-v^{2}\right)}{E}
$$




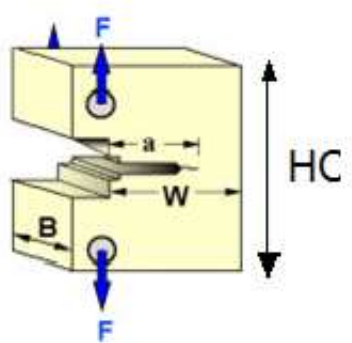

(a)

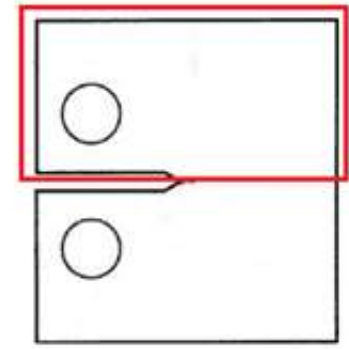

(b)

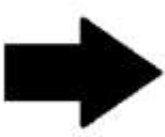

(c)

Figura 2: Corpo de prova compacto CT: (a) dimensões segundo norma ASTM E399 (1984); (b) Modelo bidimensional considerando estado plano de deformações [26], e: (c) malha simulando 1/2 do modelo bidimensional, aproveitando-se de sua simetria.

\subsection{Coleta de dados dos gráficos numéricos para o ensaio de indentação}

Para todas as simulações foram gerados gráficos da carga do indentador em função do seu deslocamento, visto que estes gráficos fornecem informações importantes para a avaliação do comportamento global durante o ciclo do ensaio. Além disso, foram gerados gráficos monitorando a distribuição numérica da deformação de trincamento (cracking strain) durante o ensaio, através do modelo de trinca difusa. Também foram gerados gráficos numéricos do comportamento dano na interface entre o filme/substrato durante o ensaio, através do modelo de delaminação, indicando possíveis falhas nesta interface.

Para gerar os gráficos da deformação de trincamento durante o ciclo de indentação, foram definidas três regiões localizadas na superfície da amostra, conforme Figura 3. A região I se localiza na superfície da amostra e próxima ao eixo de simetria. Nesta região devem acontecer as maiores tensões devido ao contato entre o indentador e a amostra [12, 14]. A região II se situa na borda de contato entre o indentador e o filme. Conforme dados encontrados na literatura, esta região apresenta uma grande concentração de trincas durante ensaios de dureza Brinell em materiais frágeis [14, 28]. Já a região III, situada fora da região de contato e que apresenta trincas devido ao gradiente de tensões de flexões que surgem nos filmes. Segundo a literatura especializada, em função da profundidade de penetração, este gradiente passaria de um campo de tensões de contato para um campo de tensões de flexão, no início do ensaio. Em seguida, a partir de uma maior profundidade de penetração do indentador, este campo de tensões no filme passaria a se comportar como tensões de membrana, ou seja, um campo de tensões de tração [14, 29].

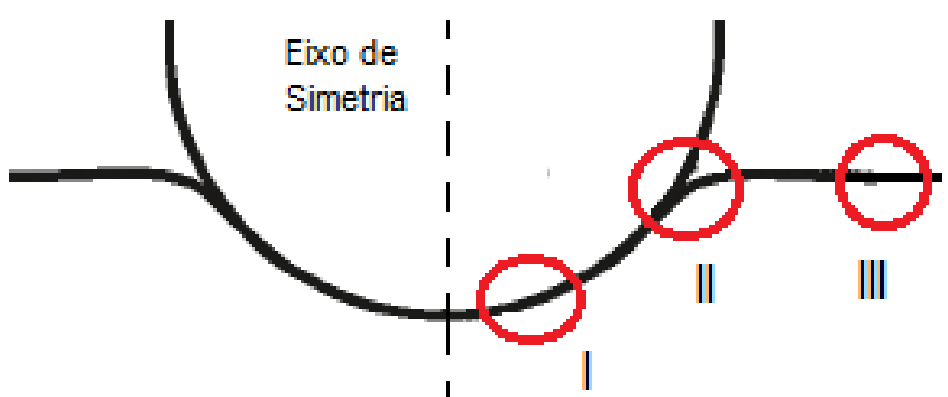

Figura 3: Localização das três regiões (I, II, III) selecionados para apresentar graficamente os valores de deformação de trincamento durante o ciclo de indentação.

Já para se obter os gráficos da distribuição numérica do dano na interface em função incremento durante o ciclo do ensaio, foram escolhidas outras três regiões (IV, V e VI), como mostradas na Figura 4. Estas regiões se localizam ao longo da interface entre o filme fino e o substrato, sendo que a região IV se situa próxima ao eixo de simetria. A região $\mathrm{V}$ fica a uma distância do eixo de simetria igual a cinco vezes a espessura do filme analisado. Por fim, a região VI se localiza a uma distância de dez vezes a espessura do filme estudado. Estas regiões foram escolhidas baseadas nos resultados numéricos de falhas por delaminação em filmes finos apresentados por Fukumasu e Souza (2015) [15]. 

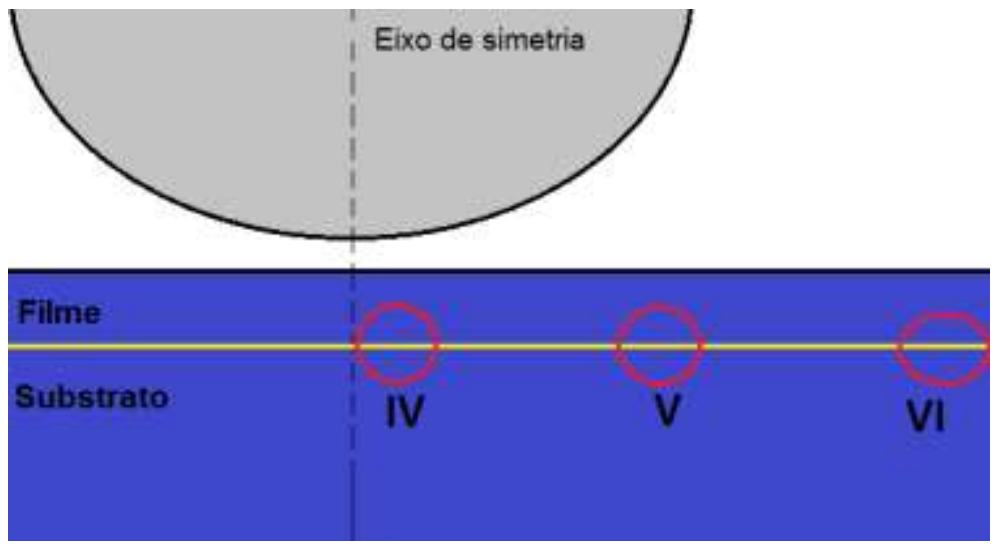

Figura 4: Localização das outras três regiões (IV, V, VI) selecionadas para fornecer graficamente valores de dano por delaminação entre o filme e o substrato.

Em 2015, Fukumasu e Souza apresentaram o parâmetro $F$, Equação (3), capaz de analisar o efeito combinado de variáveis como a carga de indentação $(P)$, o raio do indentador $(R)$ e a tenacidade à fratura do filme $\left(K_{I C}\right)$, bem como uma relação inversa entre o módulo de elasticidade do filme $(E)$ e a espessura do filme $(t)$. Além disso, segundo estes autores, o parâmetro $F_{1}$ também apresenta uma relação inversa com a área de contato $(A)$, calculada diretamente a partir do software de elementos finitos, considerando o comprimento dos elementos que estariam em contato como penetrador na profundidade máxima de indentação. Para estes autores, $F_{1}$ igual a dois representaria um limiar de significativo interesse tecnológico, sobre o qual não havia observação de propagação de trinca coesiva em sistemas compostos de filme/substrato. No presente trabalho também foi calculado este parâmetro $F 1$ e verificado a sua relação com os processos de fratura ocorridos nos ensaios simulados [15].

$$
F_{1}=\frac{P R^{2} K_{I C}^{3 / 2}}{A E \sqrt{t}}
$$

\section{RESULTADOS}

Inicialmente, simulou-se o ensaio com corpo de prova compacto para o $C r A l N$ com o objetivo de se estimar a sua energia coesiva $\left(G_{I C}\right)$. Como era de se esperar, os resultados numéricos identificaram a ponta da trinca como uma região crítica utilizando a metodologia desenvolvida por Chaves Filho et. al. (2015) para estimar a tenacidade à fratura em materiais frágeis, Figura 5 [27]. Na sequência, encontrou-se o valor para a tenacidade a fratura para o $C r A l N$ que foi de $K I C=0,87 \mathrm{MPa} \sqrt{\mathrm{m}}$. Este valor se encontra compatível com valores de tenacidade à fratura de materiais frágeis [1]. Aplicando este valor de tenacidade na Equação (2) se obteve a estimativa do da energia coesiva do filme de CrAlN a ser usado neste trabalho. Esta metodologia se mostrou mais confiável do que o procedimento encontrado em alguns trabalhos publicados como, por exemplo, no artigo de Fukumasu e Souza (2015). Pois, estes autores estimaram uma tensão máxima no filme como parâmetro para aplicar a lei de tração-separação do modelo de delaminação [15, 27].

A Tabela 3 mostra os resultados numéricos para a carga máxima do ensaio de indentação para todas as simulações realizadas. Ou seja, os dados das quatro diferentes espessuras do filme CrAlN combinado com as cinco profundidades de penetração dos ensaios realizados. Os valores obtidos comprararam os resultados da simulação das três diferentes combinações dos dois modelos de dano utilizados. Na primeira coluna, têmse os resultados da carga máxima de indentação para apenas o modelo de delaminação. Na segunda coluna, mostram-se os resultados da carga máxima com apenas o modelo de trinca difusa ativado. Na última coluna, apresentam-se os resultados da carga com os dois modelos de dano, delaminação e trinca difusa, ativados. Analisando estes resultados, nota-se que em todas as colunas, os valores numéricos da carga máxima de indentação apresentam pouca variação entre as três diferentes combinações utilizadas. Exceto, para os casos de filmes espesso e grandes profundidades de penetração. 

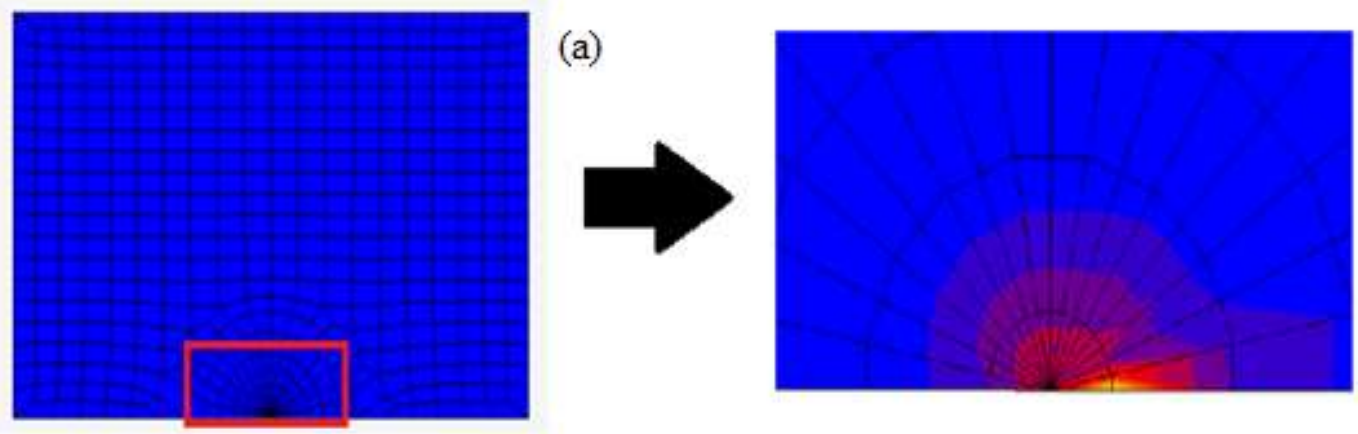

(b)

Figura 5: (a) Demarcação na malha na região da ponta da trinca do ensaio CT; (b) Imagem ampliada da área crítica na ponta da trinca mostrando as franjas do campo de tensões.

Tabela 3: Carga máxima de indentação para todas as simulações, considerando as três diferentes combinações com os dois modelos danos utilizados.

\begin{tabular}{|c|c|c|c|c|}
\hline \multirow{2}{*}{$\begin{array}{l}\text { Espessura do } \\
\text { filme }(t)\end{array}$} & \multicolumn{4}{|c|}{ CARGA MÁXIMA DE INDENTAÇÃO } \\
\hline & $h_{\max }$ & Delaminação & Trinca difusa & Delaminação + trinca difusa \\
\hline \multirow{5}{*}{$2,0 \mu \mathrm{m}$} & $10 \% t$ & $7,01 \mathrm{~N}$ & $7,04 \mathrm{~N}$ & $7,01 \mathrm{~N}$ \\
\hline & $20 \% t$ & $19,68 \mathrm{~N}$ & $19,71 \mathrm{~N}$ & $19,68 \mathrm{~N}$ \\
\hline & $50 \% t$ & $48,62 \mathrm{~N}$ & $48,65 \mathrm{~N}$ & $48,62 \mathrm{~N}$ \\
\hline & $70 \% t$ & $63,56 \mathrm{~N}$ & $63,54 \mathrm{~N}$ & $63,54 \mathrm{~N}$ \\
\hline & $100 \% t$ & $81,56 \mathrm{~N}$ & $81,96 \mathrm{~N}$ & $81,52 \mathrm{~N}$ \\
\hline \multirow{5}{*}{$3,0 \mu \mathrm{m}$} & $10 \% t$ & $14,10 \mathrm{~N}$ & $14,13 \mathrm{~N}$ & $14,10 \mathrm{~N}$ \\
\hline & $20 \% t$ & $31,07 \mathrm{~N}$ & $31,11 \mathrm{~N}$ & $31,08 \mathrm{~N}$ \\
\hline & $50 \% t$ & $67,42 \mathrm{~N}$ & $67,45 \mathrm{~N}$ & $67,43 \mathrm{~N}$ \\
\hline & $70 \% t$ & $84,60 \mathrm{~N}$ & $85,24 \mathrm{~N}$ & $84,54 \mathrm{~N}$ \\
\hline & $100 \% t$ & $104,20 \mathrm{~N}$ & $105,19 \mathrm{~N}$ & $104,38 \mathrm{~N}$ \\
\hline \multirow{5}{*}{$6,0 \mu \mathrm{m}$} & $10 \% t$ & $33,73 \mathrm{~N}$ & $33,76 \mathrm{~N}$ & $33,73 \mathrm{~N}$ \\
\hline & $20 \% t$ & $59,29 \mathrm{~N}$ & $59,31 \mathrm{~N}$ & $59,29 \mathrm{~N}$ \\
\hline & $50 \% t$ & $108,20 \mathrm{~N}$ & $108,56 \mathrm{~N}$ & $108,58 \mathrm{~N}$ \\
\hline & $70 \% t$ & $130,60 \mathrm{~N}$ & $132,24 \mathrm{~N}$ & $130,06 \mathrm{~N}$ \\
\hline & $100 \% t$ & $160,10 \mathrm{~N}$ & $63,76 \mathrm{~N}$ & $159,65 \mathrm{~N}$ \\
\hline \multirow{5}{*}{$9,0 \mu \mathrm{m}$} & $10 \% t$ & $51,46 \mathrm{~N}$ & $51,40 \mathrm{~N}$ & $51,46 \mathrm{~N}$ \\
\hline & $20 \% t$ & $83,48 \mathrm{~N}$ & $83,50 \mathrm{~N}$ & $83,48 \mathrm{~N}$ \\
\hline & $50 \% t$ & $140,50 \mathrm{~N}$ & $143,64 \mathrm{~N}$ & $140,10 \mathrm{~N}$ \\
\hline & $70 \% t$ & $170,70 \mathrm{~N}$ & $173,81 \mathrm{~N}$ & $170,22 \mathrm{~N}$ \\
\hline & $100 \% t$ & $209,10 \mathrm{~N}$ & $212,25 \mathrm{~N}$ & $208,36 \mathrm{~N}$ \\
\hline
\end{tabular}

Todos estes resultados de carga máxima de indentação, Tabela 3, se mostraram semelhantes aos encontrados na literatura para ensaios em sistemas similares [12, 14, 21]. Vale a pena ressaltar que, por exemplo, nas simulações do trabalho de Araújo e Dias (2013), o atrito foi considerado diferente de zero [21]. Do ponto de vista dos resultados de carga de indentação, para o mesmo sistema e utilizando as mesmas profundidades de penetração, os resultados encorajaram a utilizar a combinação do modelo de delaminação com o modelo de trinca difusa. O primeiro com o objetivo de avaliar as falhas adesivas (na interface) e o segundo 
capaz de mostrar as falhas coesivas. Por isso, todos os demais resultados a serem apresentados foram obtidos das simulações contendo a combinação destes dois modelos de danos.

A Figura 6 mostra um gráfico com o resultado da simulação do comportamento da carga de indentação $(P)$ em função da profundidade de penetração $(h)$ para o sistema filme/substrato estudado, para um filme com espessura de 2,0 $\mu \mathrm{m}$ submetido a uma profundidade máxima de 1,0 $\mu \mathrm{m}(50 \% t)$. Esta curva $P x h$ mostra todo o ciclo de indentação, ou seja, a etapa de carregamento do ensaio seguido de seu descarregamento, com uma deformação permanente na amostra ao final do ensaio. Neste gráfico, a carga máxima de indentação foi de 48,6 N, em conformidade com os informados na Tabela 3. Em todas as demais simulações executadas, os gráficos de Pxh apresentaram um comportamento global semelhante ao da Figura 6, porém, quanto maior a profundidade de penetração ou a espessura do filme, maior foram os valores de carga máxima de indentação e maior a área desta curva, ou seja, maior sua deformação plástica.

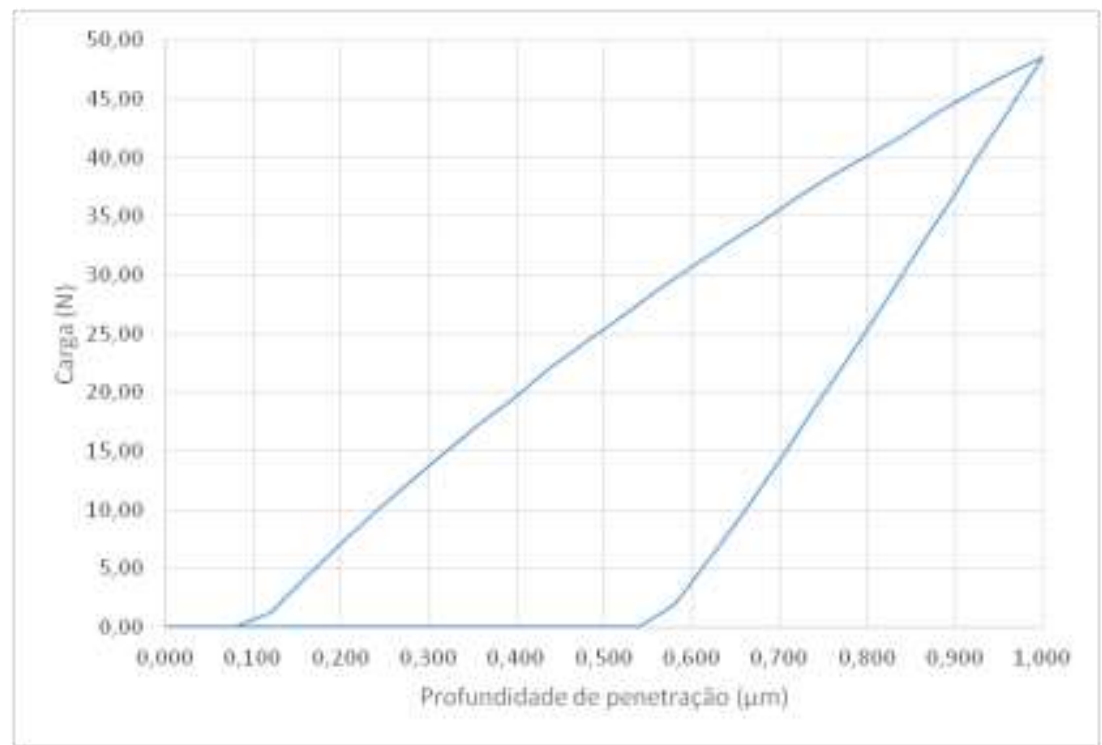

Figura 6: Curva $P x h$ do ensaio de indentação com $h_{m a ́ x}=50 \%$ t para o filme $C r A l N$ de $2,0 \mu \mathrm{m}$.

O gráfico da Figura 7 mostra outro comportamento da curva Pxh, neste caso para um sistema com filme de espessura $9 \mu \mathrm{m}$ e uma profundidade de penetração igual a sua espessura $(100 \% t)$. Seu comportamento global foi semelhante ao da Figura 6, mas sua carga máxima de indentação foi de 208,36 N e apresentou uma maior deformação permanente. A região destacada neste gráfico mostra uma descontinuidade na curva de carregamento do ensaio, indicando a ocorrência de trincas coesivas durante o ensaio [15, 30]. Contudo, mesmo obtendo estas curvas Pxh a partir de uma simulação numérica, onde se tem um maior controle do número de incrementos e uma maior possibilidade de repetitividade do ensaio, nem sempre é possível identificar as descontinuidades (trincamento) nestas curvas. Por isso, a Tabela 4 mostra qualitativamente em quais simulações ocorreu o surgimento de danos durante o ciclo de indentação, seja este dano ativado pelo modelo de delaminação ou pelo modelo de trinca difusa (deformação de trincamento) ou, ainda, por ambos.

Tabela 4: Identificação da ocorrência de danos nos diferentes ensaios simulados.

\begin{tabular}{|c|c|c|c|c|}
\hline \multirow{2}{*}{$\begin{array}{l}\text { Profundidade de } \\
\text { penetração }\end{array}$} & \multicolumn{4}{|c|}{ Espessura do filme $(t)$} \\
\hline & $2,0 \mu \mathrm{m}$ & $3,0 \mu \mathrm{m}$ & $6,0 \mu \mathrm{m}$ & $9,0 \mu \mathrm{m}$ \\
\hline$h_{\max }=0,10 t$ & sem dano & sem dano & sem dano & sem dano \\
\hline$h_{\max }=0,20 t$ & sem dano & sem dano & sem dano & sem dano \\
\hline$h_{\max }=0,50 t$ & sem dano & Trinca adesiva & Trinca adesiva & $\begin{array}{c}\text { Trincas superficial } \\
\text { e adesiva }\end{array}$ \\
\hline$h_{\max }=0,70 t$ & $\begin{array}{c}\text { Trincas superficial } \\
\text { e adesiva }\end{array}$ & $\begin{array}{c}\text { Trincas superficial } \\
\text { e adesiva }\end{array}$ & $\begin{array}{c}\text { Trincas superficial } \\
\text { e adesiva }\end{array}$ & $\begin{array}{c}\text { Trincas superficial } \\
\text { e adesiva }\end{array}$ \\
\hline$h_{\max }=t$ & $\begin{array}{c}\text { Trincas superficial } \\
\text { e adesiva }\end{array}$ & $\begin{array}{c}\text { Trincas superficial } \\
\text { e adesiva }\end{array}$ & $\begin{array}{c}\text { Trincas superficial } \\
\text { e adesiva }\end{array}$ & $\begin{array}{c}\text { Trincas superficial } \\
\text { e adesiva }\end{array}$ \\
\hline
\end{tabular}


Conforme mostrado nesta Tabela 4, para o filme $C r A l N$ com 2,0 $\mu \mathrm{m}$ de espessura ensaiado com uma profundidade de penetração de $70 \% t$, ocorreu pelo menos um processo de fratura durante o ensaio. A Figura 8(a) identificou o surgimento de uma trinca superficial próxima a bordo da impressão do indentador esférico (região II). O surgimento desta trinca superficial e o seu crescimento estão ilustrados na Figura 8(b). Ou seja, a nucleação desta trinca ocorreu próxima ao final da fase de carregamento do ensaio e seu crescimento se estendeu até o final do ensaio. O surgimento de trinca superficial na região II foi novamente verificado neste sistema com filme de $2,0 \mu \mathrm{m}$ e com uma profundidade de penetração de $100 \%$ da sua espessura. Neste ensaio com a maior profundidade de penetração, esta trinca superficial surgiu aproximadamente na metade da fase de carregamento do ensaio. As simulações encontradas na literatura também mostram a região da borda do contato entre o indentador e a amostra como crítica para o aparecimento de trincas superficiais $[12,15,21]$.

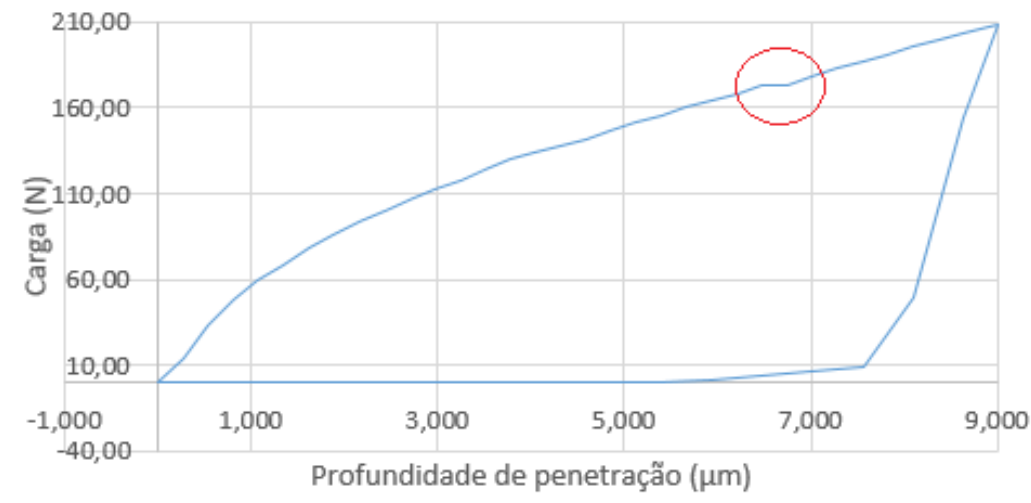

Figura 7: Curva $P x h$ do ensaio de indentação com $h_{m a ́ x}=100 \%$ t para o filme $C r A l N$ de $9 \mu \mathrm{m}$.

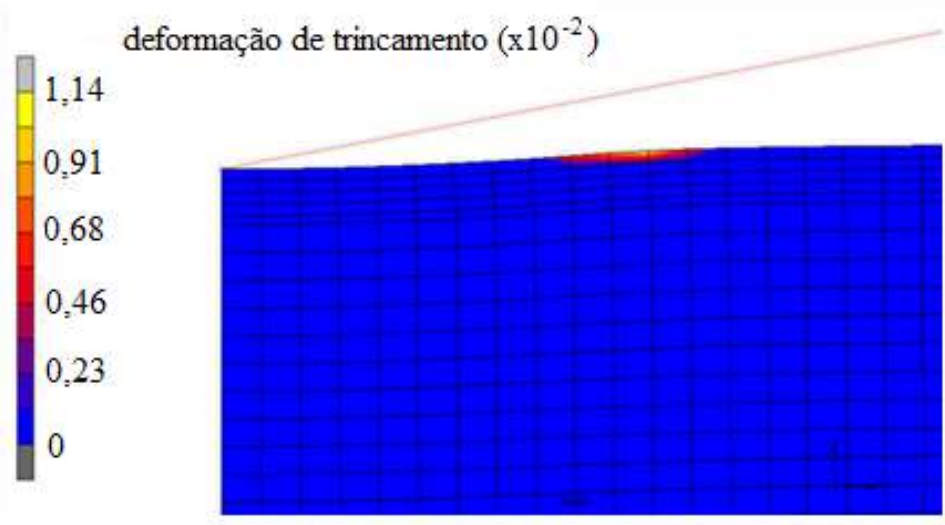

(a)

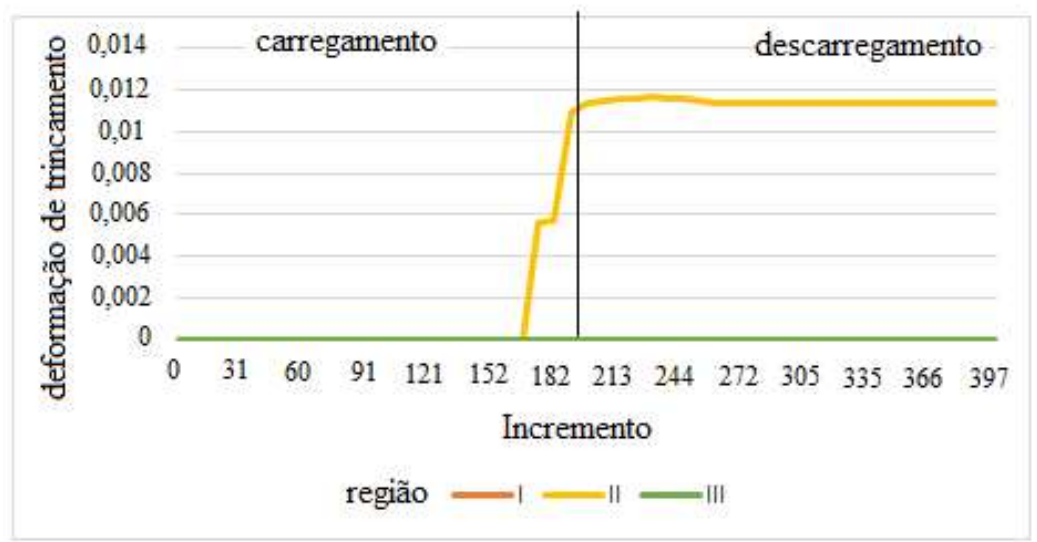

(b)

Figura 8: (a) Trinca superficial na região II no ensaio com uma profundidade de penetração de $70 \%$ do filme de $2 \mu \mathrm{m}$ de CrAlN; (b) Nucleação e crescimento desta trinca superficial durante o ciclo de indentação. 
A Figura 9 mostra os resultados numéricos obtidos pelo modelo de delaminação no ensaio de indentação para este filme $C r A l N$ com 2,0 $\mu$ m de espessura e profundidade de penetração de $70 \% t$. Na Figura 9(a) identifica-se o aparecimento de trinca adesiva na interface entre o filme e o substrato (região V). A Figura 9(b) mostra que esta trinca adesiva surgiu bem ao final da fase de carregamento do ensaio apenas na região $\mathrm{V}$ da malha, mas este dano não acarretou a uma delaminação total da interface. Este comportamento também se repetiu nos resultados para o filme com $2,0 \mu \mathrm{m}$ e com profundidade de penetração de $100 \% t$. Porém, para esta maior profundidade de penetração, o dano nesta região $\mathrm{V}$ se iniciou antes do final do carregamento do ensaio de indentação.

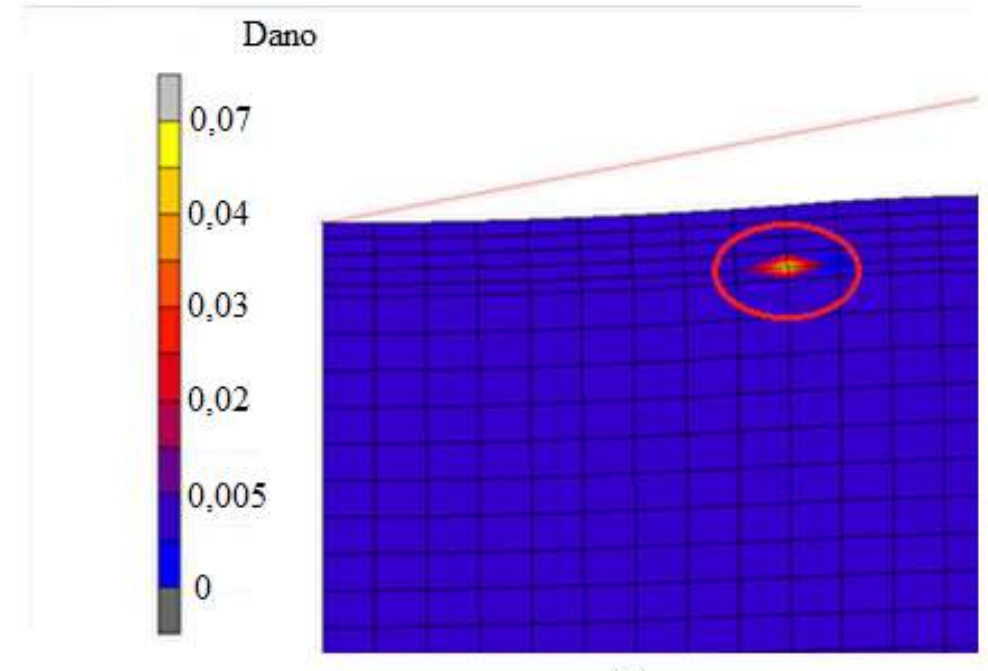

(a)

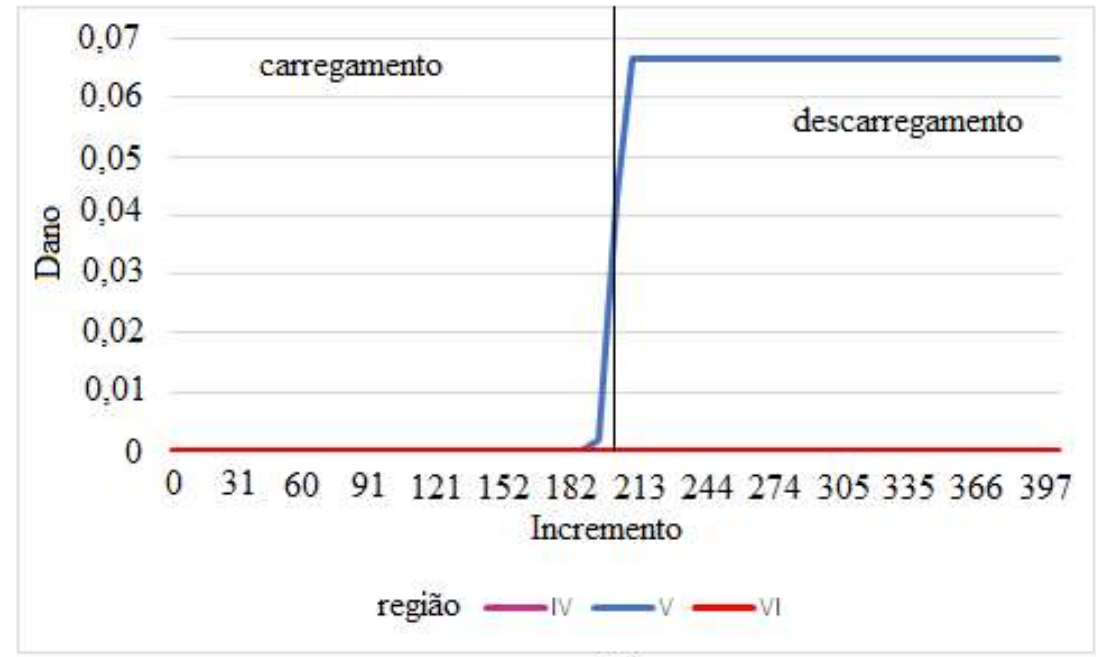

(b)

Figura 9: (a) Delaminação na região V no ensaio de indentação com uma profundidade de penetração de $70 \%$ do filme de $2 \mu \mathrm{m}$ de $\mathrm{CrAlN}$; (b) Crescimento da região delaminada durante o ciclo de indentação.

Diferente dos resultados para o filme mais fino, nas simulações com o filme $C r A l N$ de $3,0 \mu \mathrm{m}$ de espessura, o modelo de trinca difusa apresentou surgimento de trincas superficiais para as profundidades de penetração acima de $70 \%$. Estas trincas superficiais surgiram na região II antes do final do carregamento. Mas, para a maior profundidade de penetração, também surgiram trincas superficiais na região III durante o descarregamento devido à acomodação das tensões residuais após o contato entre o indentador e a amostra. Já o modelo de delaminação mostrou trincas adesivas para as profundidades de penetração acima de $50 \%$ da espessura do filme. As trincas adesivas também apareceram durante o carregamento do ensaio na região $\mathrm{V}$, porém, durante o descarregamento do ensaio, esta trinca adesiva se propagou para as regiões IV e VI sugerindo um descolamento total do filme com o substrato, especialmente no ensaio com a maior profundidade de penetração. 
Nas simulações com o filme $C r A l N$ com $6,0 \mu \mathrm{m}$ de espessura, novamente se verificou que o aumento da carga no indentação com o aumento da profundidade de penetração (Tabela 4). Também nestas simulações com o filme de $6,0 \mu \mathrm{m}$, apareceram trincas superficiais para as profundidades de penetração acima de $70 \%$. As trincas adesivas apareceram para as profundidades de penetração acima de $50 \%$ da espessura do filme. A Figura 10(a) mostra a distribuição numérica da deformação de trincamento durante o ciclo de indentação para a profundidade de penetração de $100 \%$ da espessura do filme de $6,0 \mu \mathrm{m}$. Para este filme, ocorreu o surgimento de trincas superficiais nas três diferentes regiões estudadas (I, II e III). Ou seja, na região ao redor do eixo de simetria do problema, na borda do contato entre o indentador e a amostra e na região um pouco afastada do contato do indentador. Todas estas trincas surgem antes do final do carregamento e continuam no descarregamento, sugerindo uma descamação do filme durante o ensaio de indentação. A Figura 10(b) mostra a nucleação de trincas adesivas para as profundidades de penetração de $100 \%$ da espessura deste filme. Estas trincas surgiram durante o carregamento do ensaio na interface em todas as nas regiões estudadas (regiões IV, V e VI). Ou seja, para a maior profundidade de penetração, o filme com 6,0 $\mu \mathrm{m}$ apresentou trincas adesivas em todas as regiões estudadas, sugerindo também um descolamento do filme ainda na fase de carregamento.

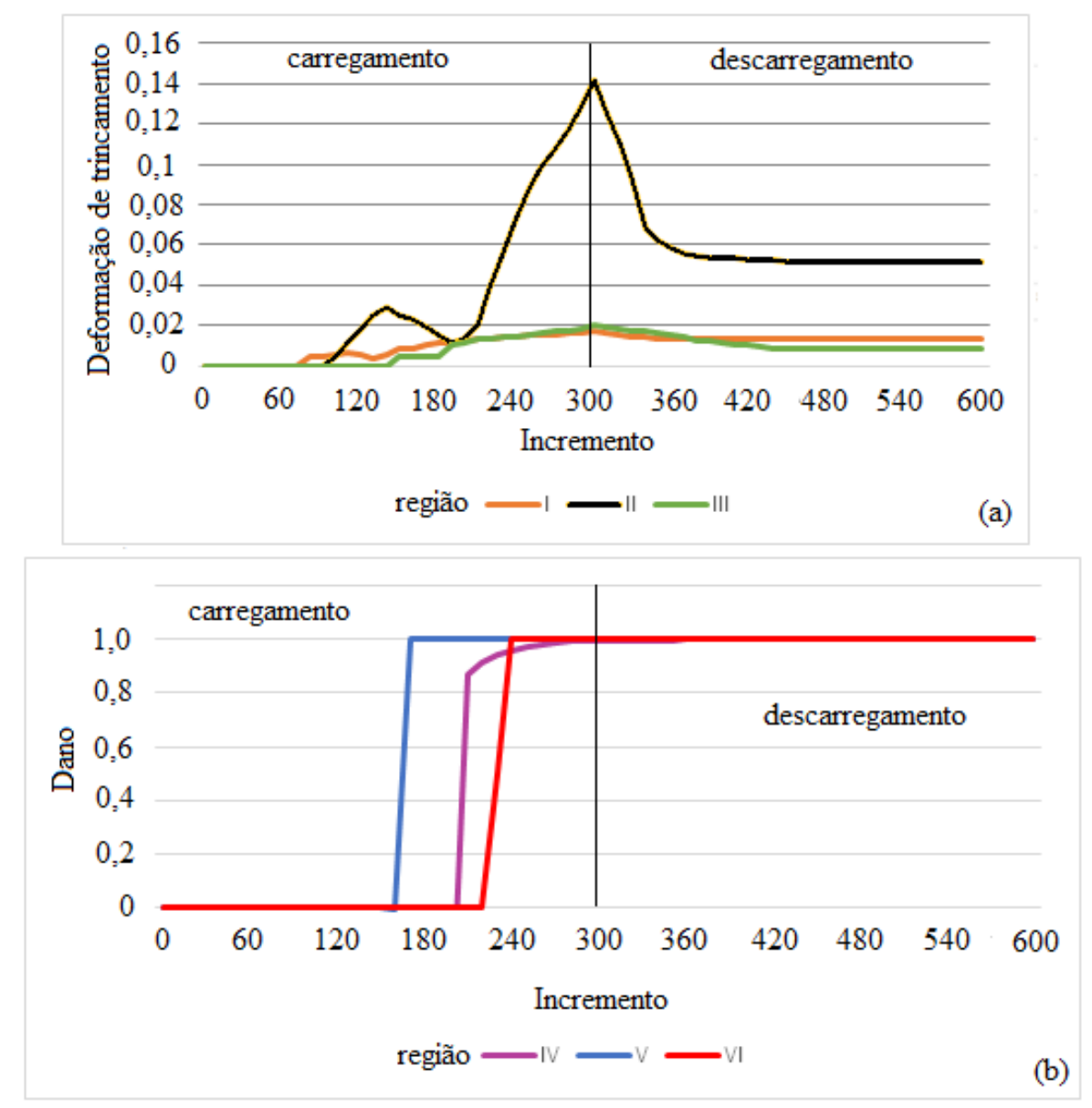

Figura 10: (a) Distribuição numérica do comportamento da deformação de trincamento para o ensaio de indentação com penetração de $100 \%$ da espessura do filme de $6,0 \mu \mathrm{m}$; (b) Distribuição numérica do dano na interface durante o mesmo ensaio.

Nas análises dos processos de dano nas simulações do sistema com o filme de maior espessura $(9,0 \mu \mathrm{m})$ tanto o modelo de trinca difusa quanto o modelo de delaminação indicaram surgimento de trincas superficiais e adesivas para as profundidades de penetração acima de $50 \%$ da espessura do filme. Os processos de fratura neste filme tiveram um comportamento semelhante ao de $6,0 \mu \mathrm{m}$, ou seja, ocorreu o surgimento de trincas superficiais nas três diferentes regiões estudadas (I, II e III) e elas surgiram antes do final do carregamento e continuaram no descarregamento, sugerindo uma descamação do filme durante o ensaio de indentação. As trincas adesivas também surgiram durante o carregamento do ensaio na interface em todas as nas regiões estudadas (regiões IV, V e VI), novamente sugerindo um descolamento do filme ainda na fase de 
carregamento.

Por fim, avaliou-se o parâmetro F1, Equação (3), com o objetivo de se avaliar o limiar do efeito combinado de diferentes variáveis (carga de indentação, o raio do indentador, tenacidade à fratura do revestimento, módulo elasticidade do filme, espessura do filme) em função do surgimento de falhas coesivas nos filmes [15]. A Figura 11(a) mostra a relação deste parâmetro com a razão entre a carga aplicada e a área da impressão $(P / A)$ para os sistemas simulados. A Figura 11(b) mostra a relação $F 1$ com a razão $h_{\text {max }} /$. Nestes gráficos, os pontos vermelhos indicam sistemas que não apresentaram falhas durante a simulação e os pontos pretos indicam os sistemas que falharam. Inicialmente, verifica-se que o valor proposto de $F 1$ igual a dois para a identificação de sistemas propensos a apresentarem falhas adesivas se aplica nos sistemas estudados. Porém, em todas as simulações realizadas com o sistema estudado, apresentaram F1 maior que o limiar igual a dois. Ou seja, o valor deste parâmetro encontrado por Fukumasu e Souza (2015) se mostrou conservativo para o sistema aqui estudado [15]. Além disso, este parâmetro F1 não deve ser aplicado nos estudos de ensaios em filmes submetidos a pequenas profundidades de penetração, como mostra a Figura 11(b).

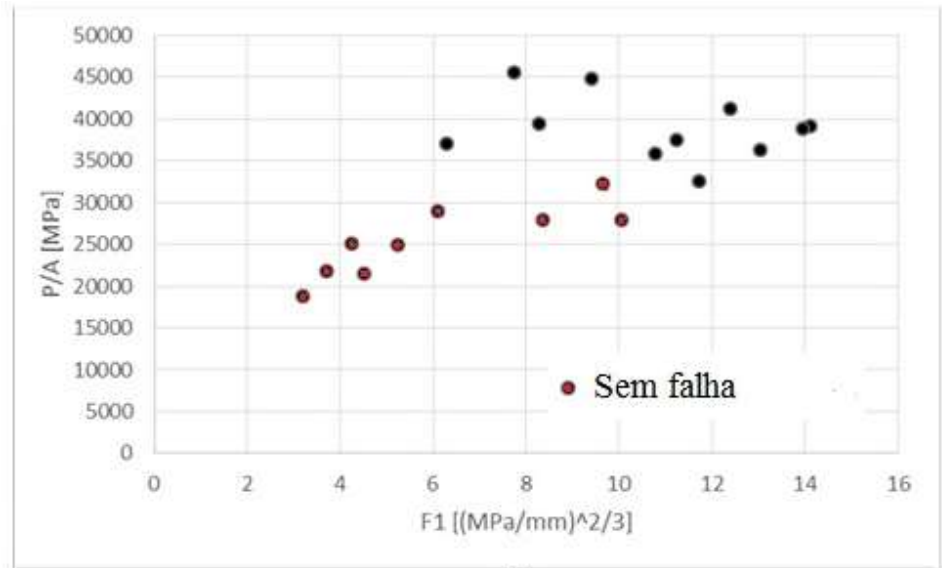

(a)

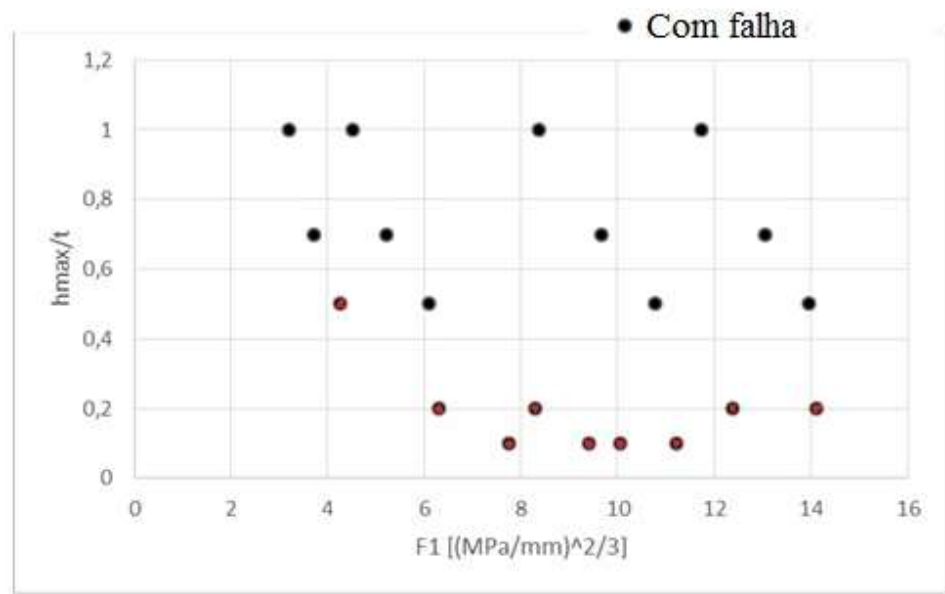

(b)

Figura 11: (a) Gráfico da razão entre a carga aplicada e a área da impressão $(P / A)$ em função de $F 1$; (b) Gráfico da razão $h_{\max } / t$ em função de $F 1$.

\section{CONCLUSÕES}

Baseando-se nos resultados obtidos pelas simulações do ensaio de indentação com penetradores esféricos através do método dos elementos finitos, foi possível concluir que a utilização concomitante de dois diferentes modelos de dano conseguiu representar o ensaio de indentação um sistema composto por um filme fino de CrAlN, com diferentes espessuras, depositados em um substrato de aço AISI 4140. Os ensaios simulados representaram bem o comportamento global do sistema estudado submetido a diferentes profundidades de penetração.

A estimativa do valor da energia Coesiva $\left(G_{I C}\right)$ do filme se mostrou consistente, pois o valor encon- 
trado estava dentro da faixa de valores comumente encontrados para materiais frágeis. Além disso, os resultados apresentados pelas simulações se mostraram coerentes com as propriedades do filme, mostrando o surgimento e crescimento de trinca na interface entre o filme e o substrato sob as mesmas condições utilizadas em outros trabalhos encontrados na literatura [14-15].

Foram avaliados os processos de fratura superficial e adesiva nestes estudos através dos dois modelos de dano implementados. Estes modelos se mostraram adequados para serem aplicados em conjunto no estudo de trincas em ensaios de indentação com indentadores esféricos, visto que eles conseguiram identificar a nucleação e o crescimento tanto de trincas coesivas quanto de trincas adesivas.

Todas as simulações do ensaio de indentação no sistema filme/substrato estudado apontaram o surgimento de trincas em todas as espessuras de filme para as maiores profundidades de penetração do indentador. Ou seja, os ensaios de indentação para os filmes com diferentes espessuras apresentaram trincas superficiais, através do modelo de trinca difusa, principalmente na região II, ou seja, no limite do contato do indentador com a amostra. Também mostraram o surgimento de trincas adesivas entre o filme e o substrato através do modelo de delaminação. Outra vantagem do presente trabalho foi mostrar a nucleação e o crescimento das trincas durante o ensaio de indentação. Contudo, ao contrário do verificado na literatura, o presente artigo mostrou que trincas adesivas ocorrem tanto na fase de carregamento como de descarregamento.

Em todas as simulações realizadas do ensaio de indentação com sistema filme $\operatorname{CrAlN}$ depositado no substrato AISI 4140, com diferentes espessuras, apareceram trincas adesivas e coesivas para as maiores profundidades de penetração. Contudo, o filme com espessura de 2,0 $\mu \mathrm{m}$ só apresentou o surgimento de trincas para os ensaios onde a profundidade de penetração foi igual ou maior que $70 \%$ da espessura do filme. Para os demais filmes, as trincas adesivas ou coesivas surgiram para a profundidade de penetração a partir de $50 \%$ da espessura do filme. Isto indicou que filmes mais espesso tem maior capacidade de suportar a cargas de indentação (dureza), porém apresentam uma menor resistência ao tricamento para grandes profundidades de penetração. Este comportamento pode estar associado às tensões de flexões que os filmes sofrem durante o ensaio de indentação com penetradores esféricos.

Este trabalho também confirmou a validade do uso do parâmetro F1 proposto por Fukumasu e Souza (2015) [15]. Porém, para o sistema estudado, o valor encontrado para este parâmetro se mostrou conservativo. Finalmente, este parâmetro não se mostrou adequado para sistemas de filmes/substratos submetidos a pequenas profundidades de penetração. Ou seja, sob baixas profundidades de penetração (10\% $t$ ), os campos de tensões e de deformação se distribuem fortemente no filme, sem acarretar falhas coesivas ou adesivas [56].

\section{AGRADECIMENTOS}

Os autores agradecem os recursos provenientes dos órgãos de fomento à pesquisa CAPES (processo BEX 6569-14) e CNPq (Universal 14/2014, processo 460943/2014-6).

\section{BIBLIOGRAFIA}

[1] CALISTER Jr, W., Ciência e Engenharia de Materiais, Uma Introdução, 8 a Ed., Rio, LTC, 2012.

[2] ZENG, K., CHIU, C., “An Analysis of Load-Penetration Curves from Instrumented Indentation”, Acta Materialia, v. 49, pp. 3539-3551, 2001.

[3] LEE, H., LEE, J. H., PHARR, G. M., “A Numerical Approach to Spherical Indentation Techniques for Material Property Evaluation”, Journal of the Mechanics and Physics of Solids, v. 53, pp. 2073-2069, 2005.

[4] FISCHER-CRIPPS, A. C., "Critical Review of Analysis and Interpretation of Nanoindentation Test Data", Surface \& Coatings Technology, v. 200, pp. 4153-4165, 2006.

[5] DIAS, A.M.S., GODOY, G.C.D, "Determination of stress-strain curve through Berkovich indentation testing”, Materials Science Forum, v. 636-637, pp. 1186-1193, 2010.

[6] LIBÓRIO, M.S., DIAS, A.M.S., SOUZA, R.M., "Determination of Film Thickness through Simulation of Vickers Hardness Testing”, Materials Research, 20 (3), pp. 755-760, 2017.

[7] HUANG, X., PELEGRI, A. A., "Finite element analysis on nanoindentation with friction contact at the film/substrate interface”, Composites Science and Technology, v. 67, pp. 1311-1319, 2007.

[8] SUN, Y., BLOYCE, A., BELL, T., "Finite Element Analysis of Plastic Deformation of Various TiN Coating/Substrate Systems under Normal Contact with a Rigid Sphere", Thin Solid Films, v. 271, pp. 122131, 1995. 
[9] SOUZA, R. M., MUSTOE, G. G. W., MOORE, J. J., "Finite Element Modeling of the Stresses, Fracture and Delamination During the Indentation of Hard Elastic Films on Elastic-Plastic Soft Substrates", Thin Solid Films, v. 392, pp. 65-74, 2001.

[10] ANTUNES, J. M., MENEZES, L. F., FERNANDES, J. V., "3-dimensional Numerical Simulation of Vickers Indentation Testing”, International Journal of Solids and Structures, v. 43, pp. 784-806, 2006.

[11] DIAS, A.M.S., MODENESI, P. J., CRISTINA, G. C., "Computer Simulation of Stress Distribution During Vickers Hardness Testing of WC-6Co”, Materials Research, v. 9, n.1, pp. 73-76, 2006.

[12] DIAS, A.M.S., SOTANI, P.F.B., GODOY, G.C. "Simulação do Ensaio de Indentação em Filmes Finos com o uso de Modelos de Trinca Difusa”, Revista Matéria, v. 15, n.3, pp. 422-430, 2010.

[13] LOTTI, R. S., MACHADO, A. W., MAZZIEIRO, E. T., et al., “Aplicabilidade científica do método dos elementos finitos”. Revista Dental Press Ortodon Ortop Facial, v. 11, n.2, pp. 35-43, 2006. Disponível em: http://www.scielo.br/pdf/\%0D/dpress/v11n2/a06v1ln2.pdf. Acesso em 2018.

[14] ARAÚJO R., DIAS A.M.S., "Numerical Evaluation of Strength in the Interface during Indentation Spherical Testing in Thin Films", Materials Science and Applications, v. 5, pp. 149-157, 2014.

[15] FUKUMASU, N. K.; SOUZA, R. M., "Numerical evaluation of cohesive and adhesive failure modes during the indentation of coated systems with compliant substrates", Surface \& Coatings Technology, v. 260, pp. 266-271, 2015.

[16] LIPING, W., ZHANG, G., WOOD, R.J.K., et al., "Fabrication of CrAlN nanocomposite films with high hardness and excellent anti-wear performance for gear application", Surface \& Coatings Technology, v. 204, pp. 3517-3524, 2010.

[17] GHRIBA, T., TLILIB, B., NOUVEAUC, C., et al., "Experimental investigation of the mechanical micro structural and thermal properties of thin CrAIN layers deposited by PVD technique for various aluminum percentages", Physics Procedia, v.2, pp. 1327-1336, 2009.

[18] BeNLATRECHE, Y., NOUVEAU, C., AKNOUCHE, H., et al., Physical and Mechanical Properties of CrAlN and CrSiN Ternary Systems of Wood Machining Applications, Wiley, InterSience, 2009.

[19] GODOY G.C.D, MANCOSU R.D., MACHADO R.R., et al., "Which hardness (nano or macrohardness) should be evaluated in cavitation?", Tribology International, v. 42, pp. 1021-1028, 2009.

[20] Marc ${ }^{\mathrm{TM}}$ MSC, Software Corporation, Volume A: Theory and User Information; User's Manual, Available from <http://www.mscsoftware.com>. Acesso em: 13/03/2018.

[21] ARAÚJO R., DIAS A.M.S., "Numerical study of the influence of friction coefficient for indentation testing in thin films", Revista Matéria, v. 18, n.1, pp. 55-66, 2013.

[22] PULECIO, S.A.R., ALCALÁ J., SOUZA, R.M., "The reduced modulus in the analysis of sharp instrumented indentation tests", Journal of Materials Research, v. 27, n.16, pp. 2148-2160, 2012.

[23] MEDEIROS, E. E., DIAS, A. M. S., CHRISTÓFORO, A. L., "Numerical Simulation of Mechanical Fracture Testing”, International Journal of Materials Engineering, v. 2, n.5, pp. 61-66, 2012.

[24] ZHU, W., YANG, L., GUO, J.W., ZHOU, Y.C., LU, C., "Determination of interfacial adhesion energies of thermal barrier coatings by compression test combined with a cohesive zone finite element model", International Journal of Plasticity, v. 64, pp.76-87, 2015.

[25] ASTM E-399, Standard Test Method for Plane Strain Fracture Toughness of Metallic Materials, 1984.

[26] ANDERSON, T.L., Fracture Mechanics, Fundamentals and Applications, third edition, New York, CRC Press, Inc., 2004.

[27] CHAVES FILHO,O. F., MEDEIROS, G. S., et al., "Simulation of the Fracture Process through a Damage Model in Different Materials", International Journal of Research and Review in Applied Science, v. 23 (3), pp.233-240, 2015.

[28] GODOY, G. C. D., MANCOSU, R. D., LIMA, M. M., et al., "Influence of plasma nitriding and PAPVD Cr1-xNx coating on the cavitation erosion resistance of an AISI 1045 steel”, Surface and Coatings Technology, v. 200, n.18-19, pp.5370-5378, 2006.

[29] VANIMISETTI, S. K., NARASIMHAN, R., “A Numerical Analysis of Spherical Indentation Response of Thin Hard Films on Soft Substrates", International Journal of Solids and Structures, v.43, pp.6180-6193, 2006.

[30] CHEN, J., BULL, S.J., “Assessment of the toughness of thin coatings using nanoindentation under dis- 
placement control", Thin Solid Films, v.494, pp. 1-7, 2006.

\section{ORCID}

Ozias Ferreira Chaves Filho

https://orcid.org/0000-0002-7367-9689

Avelino Manuel da Silva Dias

https://orcid.org/0000-0001-8974-5137 